\title{
$A b$ initio prediction of high-temperature magnetic relaxation rates in single-molecule magnets
}

Daniel Reta, Jon G. C. Kragskow and Nicholas F. Chilton*

Department of Chemistry, School of Natural Sciences, The University of Manchester, Oxford Road, Manchester, M13 9PL, UK.

\begin{abstract}
Organometallic molecules based on $\left[\mathrm{Dy}\left(\mathrm{Cp}^{\mathrm{R}}\right)_{2}\right]^{+}$cations have emerged as clear frontrunners in the search for high-temperature single-molecule magnets. However, despite a growing family of structurally-similar molecules, these molecules show significant variations in their magnetic properties, demonstrating the importance of understanding magnetostructural relationships towards developing more efficient design strategies. Here we refine our $a b$ initio spin dynamics methodology and show that it is capable of quantitative prediction of relative relaxation rates in the Orbach region. Applying it to all reported $\left[\mathrm{Dy}\left(\mathrm{Cp}^{\mathrm{R}}\right)_{2}\right]^{+}$cations allows us to tease out differences in their relaxation dynamics, highlighting that the main discriminant is the magnitude of the crystal field splitting. We subsequently employ the method to predict relaxation rates for a series of hypothetical organometallic sandwich compounds, revealing an upper limit to the effective barrier to magnetic relaxation of around $2200 \mathrm{~K}$, which has been reached. However, we show that further improvements to single-molecule magnets can be made by moving vibrational modes off-resonance with electronic excitations.
\end{abstract}

\section{Introduction}

The ultimate miniaturisation of classical memory devices lies in the use of atoms or molecules to store binary data. ${ }^{1}$ Single-molecule magnets (SMMs), molecules that exhibit slow magnetic relaxation and memory effects, provide a flexible platform for realising high-density data storage. The first single-molecule magnet $\left\{\mathrm{Mn}_{12}\right\}$ was shown to display magnetic hysteresis and a magnetic reversal (or magnetic relaxation) rate that is exponentially dependent on temperature above $2.5 \mathrm{~K},{ }^{2}$ which is the hallmark of thermally-activated relaxation over an intrinsic energy barrier $\left(U_{\text {eff }}\right){ }^{3}$ The relaxation process was theoretically elaborated and confirmed as a concatenated series of single spin-phonon transitions known as the Orbach process (often called a multi-phonon process) allowing the SMM to traverse its excited spin states and reverse its magnetization. ${ }^{4-6}$ The operation of SMMs is thus inextricably linked to 
their electronic structure, highlighting the crucial role of magnetic anisotropy in producing an energy barrier for magnetic relaxation. With the discovery that monometallic lanthanide complexes could also show SMM behaviour, ${ }^{7,8}$ a conceptual shift in SMM design took place. Owing to the radially-contracted $4 \mathrm{f}$ orbitals and nearly unquenched orbital momentum of the trivalent lanthanides, simple electrostatic considerations gave design criteria to achieve large anisotropy; ${ }^{9-12}$ for instance linear coordination geometry for Dy(III), or equatorial coordination geometries for $\operatorname{Er}(\mathrm{III})$. This has driven a huge increase in $U_{\text {eff }}$ barriers ${ }^{13}$ and pushed the singlephonon-driven Orbach process to higher temperatures, often replaced by a two-phonon Raman process dominating below ca. $50 \mathrm{~K} .{ }^{14}$ An important component of larger $U_{\text {eff }}$ barriers is the presence of larger energy gaps between electronic excited states (i.e. stronger crystal field splitting), making it is far from obvious that the same low-energy phonons (lattice acoustic modes) should be responsible for effecting magnetic relaxation as was proposed for $\left\{\mathrm{Mn}_{12}\right\}$. Fortunately, recent theoretical efforts have begun to establish robust and systematic methodologies to treat these problems, ${ }^{15-21}$ targeting a new approach of engineering spinphonon coupling.

The most successful class of SMMs thus far have converged to a series of Dy(III)-based metallocenium cations ${ }^{19,22,23}$ (Figure 1, left): $\quad\left[\mathrm{Dy}\left(\mathrm{Cp}^{\mathrm{iPr} 4}\right)_{2}\right]\left[\mathrm{B}\left(\mathrm{C}_{6} \mathrm{~F}_{5}\right)_{4}\right] \quad(\mathbf{1}){ }^{22}$ $\left[\mathrm{Dy}\left(\mathrm{Cp}^{\mathrm{ttt}}\right)_{2}\right]\left[\mathrm{B}\left(\mathrm{C}_{6} \mathrm{~F}_{5}\right)_{4}\right](\mathbf{2}),{ }^{19}\left[\mathrm{Dy}\left(\mathrm{Cp}^{\mathrm{iPr} 5}\right)_{2}\right]\left[\mathrm{B}\left(\mathrm{C}_{6} \mathrm{~F}_{5}\right)_{4}\right] \quad(\mathbf{3}),{ }^{22}\left[\mathrm{Dy}\left(\mathrm{Cp}^{\mathrm{iPr} 4 \mathrm{Et}}\right)_{2}\right]\left[\mathrm{B}\left(\mathrm{C}_{6} \mathrm{~F}_{5}\right)_{4}\right] \quad(\mathbf{4}),{ }^{22}$ $\left[\mathrm{Dy}\left(\mathrm{Cp}^{\mathrm{iPr} 4 \mathrm{Me}}\right)_{2}\right]\left[\mathrm{B}\left(\mathrm{C}_{6} \mathrm{~F}_{5}\right)_{4}\right](\mathbf{5})^{22}$ and $\left[\mathrm{Dy}\left(\mathrm{Cp}^{\mathrm{iPr} 5}\right)\left(\mathrm{Cp}^{*}\right)\right]\left[\mathrm{B}\left(\mathrm{C}_{6} \mathrm{~F}_{5}\right)_{4}\right](\mathbf{6}){ }^{23}$ These complexes are chemically very similar to one-another as they only differ in the cyclopentadienyl (Cp) substituents and even share the same $\left[\mathrm{B}\left(\mathrm{C}_{6} \mathrm{~F}_{5}\right)_{4}\right]^{-}$counterion, though they do crystallise in different space groups: $P_{2_{1}}, P_{\overline{1}}, P_{2_{1} / n}, P_{2_{1} / n}, P_{2_{1} / c}$ and $P_{2_{1} / c}$, for 1-6 respectively. Despite their similarity, these compounds display a significant variation in their magnetic relaxation rates (Figure 1, right). Our numbering scheme is chosen to reflect the ordering of their $100 \mathrm{~s}$ blocking temperatures (the temperature at which the relaxation time is $100 \mathrm{~s}$, herein $\mathrm{T}_{\mathrm{B}, 100 \mathrm{~s}}$ ), and while there are some crossovers in different temperature regimes, overall $\mathbf{1}$ is the fastest, $\mathbf{6}$ is the slowest, and 2-5 are very similar. Indeed, considering estimated standard deviations (ESDs) for the experimental relaxation rates shows that, within error, the relaxation rates in the Orbach region for 3-5 cannot be distinguished (Figure S1). ${ }^{24}$ Due to their differing relaxation rates, their $\mathrm{T}_{\mathrm{B}, 100 \mathrm{~s}}$ values span almost $50 \mathrm{~K} ; \mathrm{T}_{\mathrm{B}, 100 \mathrm{~s}}$ lies well within the Raman regime for $\mathbf{1}$, while it falls at the intersection between the Raman and Orbach regimes for $\mathbf{2 - 5}$, and is at the start of the Orbach regime for 6. Thus, 1-6 are an ideal set of compounds to unpick how subtle chemical 
differences result in such different magnetic relaxation rates, and, ideally, to establish the route forward to even better performing SMMs.

In this paper we refine our ab initio method for spin-dynamics ${ }^{19,21,25}$ and calculate the relaxation dynamics of 1-6 to determine what causes the differences in their dynamic magnetic properties. We show that our methodology is capable of quantitative prediction of relative rates of magnetic relaxation, subject to a $c a$. ten-fold overestimation with respect to experiment, giving us confidence in using the approach to compare the underlying spin-phonon coupling in the Orbach region. Using a vibrational-mode-weighted-decomposition of the relaxation rate matrices, we find that the largest discriminant in the magnetic relaxation rates between $\mathbf{1}$ and $\mathbf{6}$ is their static electronic structures; that is, the energy gaps are largest for $\mathbf{6}$ and smallest for 1. This confirms initial suggestions that the shorter Dy-Cp distances in $\mathbf{6}$ are responsible for its record-breaking properties. However, there is a limit to how large the CF splitting can be, and hence, we perform spin-dynamics calculations on theoretical bis- $\mathrm{Cp}^{\mathrm{R}} / \mathrm{Cb}^{\mathrm{R}}-\mathrm{Dy}$ (III) $\mathrm{SMMs}$ (where $\mathrm{Cb}$ is cyclobutadienyl) to show that $i$ ) energy barriers are unlikely to be increased much beyond $U_{\text {eff }}=2217(16) \mathrm{K}$ for $\mathbf{6}$, and $\left.i i\right)$ yet slower relaxation rates can be achieved by reducing the resonance between vibrational modes and electronic states; for instance, an isolated $\left[\mathrm{Dy}\left(\mathrm{C}_{5} \mathrm{Me}_{5}\right)_{2}\right]^{+}$cation is predicted to have relaxation rates four orders of magnitude slower than 6, despite having a smaller $U_{\text {eff }}$ barrier.
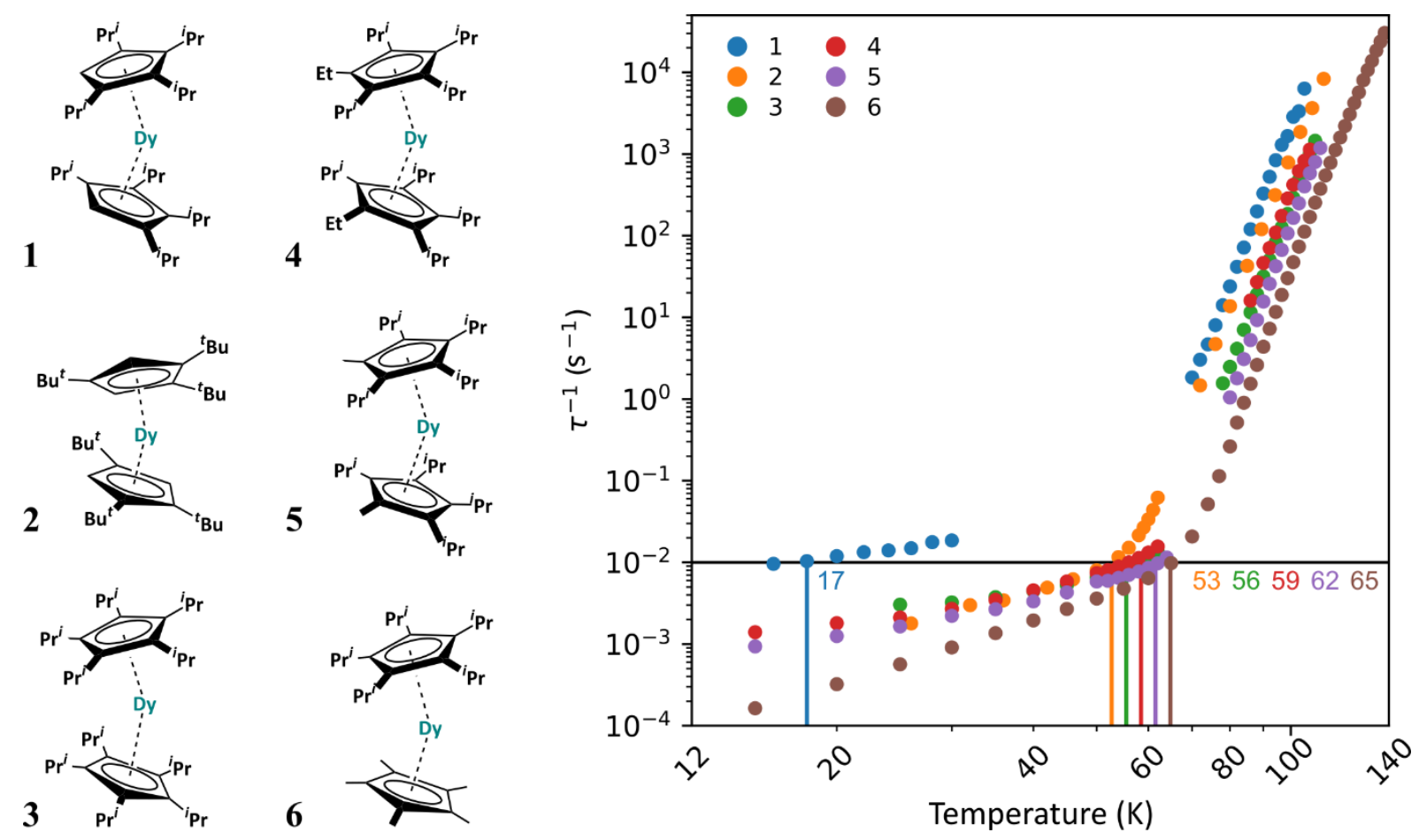

Figure 1. (Left) Schematic representation of the cations in 1-6. (Right) Magnetic relaxation rates for 1-6 ordered by $\mathrm{T}_{\mathrm{B}, 100 \mathrm{~s}}$. 


\section{Methods}

Our approach for modelling the spin dynamics of Dy(III)-based SMMs has been given in detail in our recent works, ${ }^{19,21,25}$ and is explained in the Supporting Information Section S4. Generally, it consists of three steps: $i$ ) calculation of the molecular vibrational modes in the gas-phase using density-functional theory (DFT); ii) calculation of spin-phonon coupling (note: our calculations are in the gas phase and hence are not truly phonons, but nonetheless we use the common terminology to reflect the experimental situation) using complete active space self-consistent field spin-orbit (CASSCF-SO) calculations; and iii) simulation of spindynamics. Unlike the first iteration of our method ${ }^{19}$ we no longer calibrate atomic displacements (as this effect is largely due to acoustic modes which are not currently included in our model), we now use a resolution of the identity method for approximation of two electron integrals in CASSCF-SO, ${ }^{25}$ and have revised our definition of zero-point displacement (Eq. S1). We also herein explore three different definitions of the spin-phonon coupling, including temperature-dependent spin-phonon coupling via temperature-dependent displacements (Eq. S2) $)^{21}$ and a first order Taylor expansion, and compare the choice of Boltzmann or BoseEinstein phonon statistics; see Supporting Information Section S4. Calculating the relaxation rates for compound $\mathbf{2}$ to assess these options, we find that the rates show no dependence on the choice of spin-phonon coupling or phonon statistics (Figures S19 and S20), and henceforth we employ Bose-Einstein statistics and a first-order Taylor expansion to calculate the spin-phonon coupling.

The gas-phase normal modes of the cations in 1-6 are calculated with $\mathrm{PBE}^{26,27}$ and $\mathrm{PBE}^{28}$ density-functionals in conjunction with Grimme's empirical dispersion correction ${ }^{29}$ within the Gaussian09 $\mathrm{d}^{30}$ suite of programs (Section $\mathrm{S} 2$ in the SI). We determine the maximal displacement along each normal mode using Boltzmann statistics of each harmonic oscillator at $100 \mathrm{~K}$, and subsequently calculate the spin-phonon couplings using CASSCF-SO within the OpenMolcas ${ }^{31}$ package (Section S4 in the SI). At the crystalline and optimised geometries, we determine the electronic structure with a state-average CASSCF calculation for the $21 S=5 / 2$ states of Dy(III) followed by non-perturbative SO coupling, and the lowest 16 states $\left({ }^{6} \mathrm{H}_{15 / 2}\right.$ multiplet) of the molecule are projected onto a crystal field (CF) Hamiltonian acting in the $2 J$ $+1\left|m_{J}\right\rangle$ basis. ${ }^{32}$ Improving the quality of the CASSCF method to include more spin states (see Table S5) makes a negligible difference to the results (Tables S6-S9 and Figures S13-S14), and 
indeed there is also negligible difference using the PBE0-optimised geometries (Figure S1718).

The spin-phonon coupling for each vibrational mode is determined from the CF decomposition of a CASSCF-SO calculation for distorted structures in the positive and negative directions along normal mode coordinates, with reference to the $\mathrm{CF}$ decomposition at the equilibrium geometry. The dependence of the CF parameters (CFPs) with distortion is fitted to a third-order polynomial (Eqn. S18), which can be used to interpolate the CFPs at a given temperature according to a temperature-dependent displacement (Eqns. S11, S16), or to determine the first derivative of the CFPs in a Taylor expansion (Eqn. S12). With this information, the perturbing CF matrices for each mode are determined in the equilibrium electronic eigenbasis and used to calculate the transition rates between all CF states. It is here that the only free parameter in our model is introduced, as a single fixed Gaussian linewidth parameter for each normal mode. In the final step, the master equation is constructed and solved to obtain the relaxation rates. ${ }^{19}$

\section{Results}

The DFT-optimised structures obtained for 1-6 are very similar to the experimentally determined crystal geometries (Table S3), where the largest RMSD (Dy atom, Cp rings, and Cp-bound $\mathrm{C}$ atoms) is $0.358 \AA$ for $\mathbf{4}$. Comparing the electronic structures between optimised and crystalline geometries, we find that the optimised geometries always show smaller energy gaps between the electronic states than the crystal geometries (Figure S15) and that the optimised geometries show overall CF splittings that correlate well with the ordering of $\mathrm{T}_{\mathrm{B}}$ (Figure 2 and S15), but that this does not hold for all compounds when considering the crystal geometries (Figure S16). We find that the main anisotropy axis of the ground Kramers doublet is well-approximated by the average Dy-C $\mathrm{p}_{\text {centroid }}$ vector, and that the ground doublet is $| \pm 15 / 2\rangle$, followed sequentially by $| \pm 13 / 2\rangle,| \pm 11 / 2\rangle,| \pm 9 / 2\rangle,| \pm 7 / 2\rangle$ and $| \pm 5 / 2\rangle$ excited doublets, while the two most energetic doublets are mixed $m_{J}$ functions (Table S6), in agreement with previous works. ${ }^{19,23}$ The energy gaps between the ground and first excited doublets (optimised geometries) are 414, 461, 478, 479, 476 and $530 \mathrm{~cm}^{-1}$ for 1-6, respectively, in good correlation with the ordering of the experimental relaxation rates; $\mathbf{1}$ is the smallest, $\mathbf{6}$ is the largest, and 2-5 are very similar. Our results for $\mathbf{6}$ are in good agreement with the original XMS-CASPT2 calculations performed for a similar optimized structure (Table S10). ${ }^{23}$ 


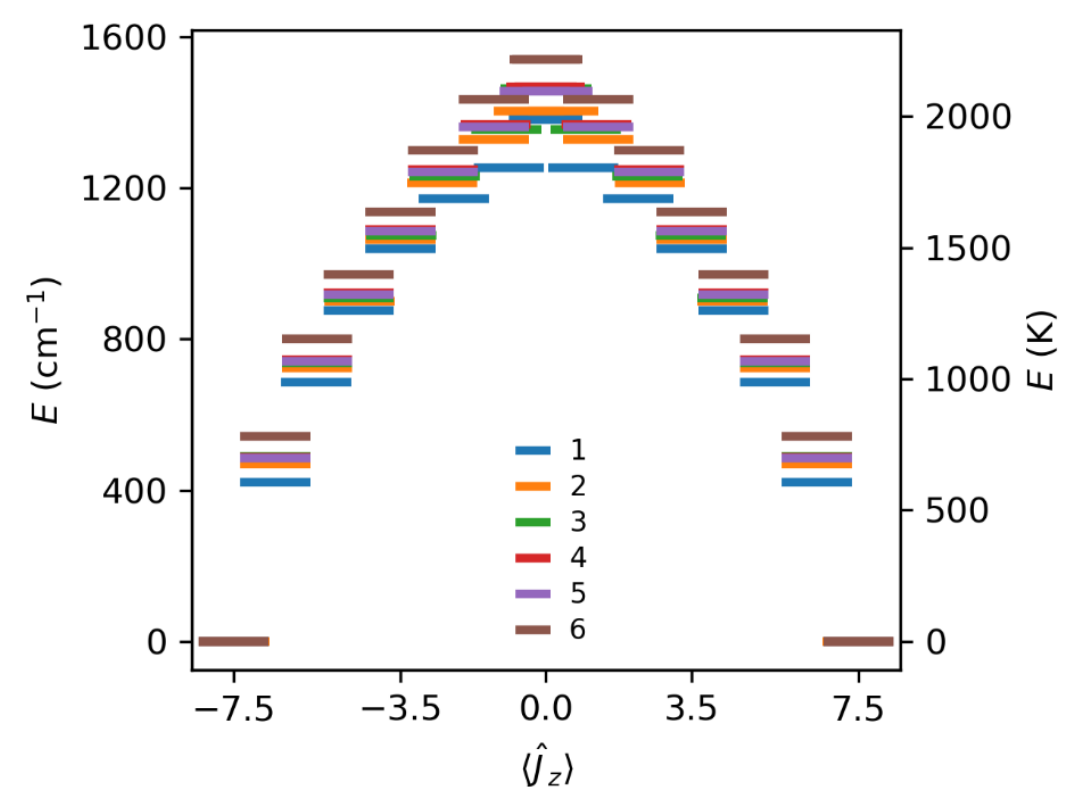

Figure 2. Comparison of the electronic structure of compounds 1-6 calculated with the crystal field parameters obtained from CASSCF-SO calculations at the PBE-optimised gas-phase geometries.

Employing our $a b$ initio spin-dynamics approach (see Methods and Supporting Information), we calculate the magnetic relaxation rates for 1-6, where the only free parameter is a constant vibrational linewidth for all modes. Here we compare full-width-half-maximum (FWHM) linewidths of 6,10 and $20 \mathrm{~cm}^{-1}$ for all compounds, which are consistent with the IR spectra (FWHM ca. $6-26 \mathrm{~cm}^{-1}$, Figures S8-S12). Calculation of the spin-dynamics for 1-6 using the PBE vibrational modes (Figure 3; results using PBE0 are nearly identical, Figure S21, hence we will only consider the PBE results further) show that calibration of the normal mode energies to IR spectra (linear calibration: slopes of $0.94-1.04$ and intercepts of $-9-+70 \mathrm{~cm}^{-1}$, Figures S8-S12, Table S4 and reference 19) is not crucial: we see the largest influences in 2 , however overall the changes are modest. For larger FWHM values, more modes come into resonance for more transitions and thus relaxation rates generally increase with linewidth (Figures 3 and S21). Crucially, however, for FWHM =6, 10 or $20 \mathrm{~cm}^{-1}$, our method predicts the correct ordering of the calculated rates: $\mathbf{1}$ is always fastest, $\mathbf{6}$ is always slowest, and $\mathbf{2 - 5}$ are very similar (Figure 4). Interestingly, in all cases we overestimate the relaxation rates by about a factor of $c a .10\left(\tau_{\operatorname{sim}} / \tau_{\text {exp }}\right.$ at $100 \mathrm{~K}$ with $\mathrm{FWHM}=10 \mathrm{~cm}^{-1}$ ranges from $5-40$ for 1-6). It is tempting to decrease the linewidth in order to match the experimental rates as closely as possible, and this would require FWHM values of $c a .1-4 \mathrm{~cm}^{-1}$ for 1-6 (Figures S22). However, for FWHM $<6 \mathrm{~cm}^{-1}$ the calculated rates no longer show the experimental ordering 
(e.g. for FWHM $=2 \mathrm{~cm}^{-1}$ Figure 4) and the profiles start to deviate significantly from those obtained with larger linewidths; thus, we suggest that results with FWHM $<6 \mathrm{~cm}^{-1}$ are not reliable (indeed such narrow linewidths are not consistent with the experimental IR spectra). While mode-energy- and temperature-dependent linewidths based on finite phonon lifetimes have been proposed by Lunghi et al., ${ }^{15}$ we have found that this is not appropriate for modelling the magnetic relaxation in bis-alkoxide Dy(III) $\mathrm{SMMs},{ }^{33}$ and this conclusion remains unchanged for the present $\left[\mathrm{Dy}\left(\mathrm{Cp}^{\mathrm{R}}\right)_{2}\right]^{+}$cations (see Figure S23 and S24). Hence, we identify that our method using the PBE density-functional without calibration and a single linewidth parameter of $c a .10 \mathrm{~cm}^{-1}$ is capable of quantitative prediction of the relative Orbach relaxation rates, subject to an overestimation of approximately one order of magnitude in comparison to experiment. 

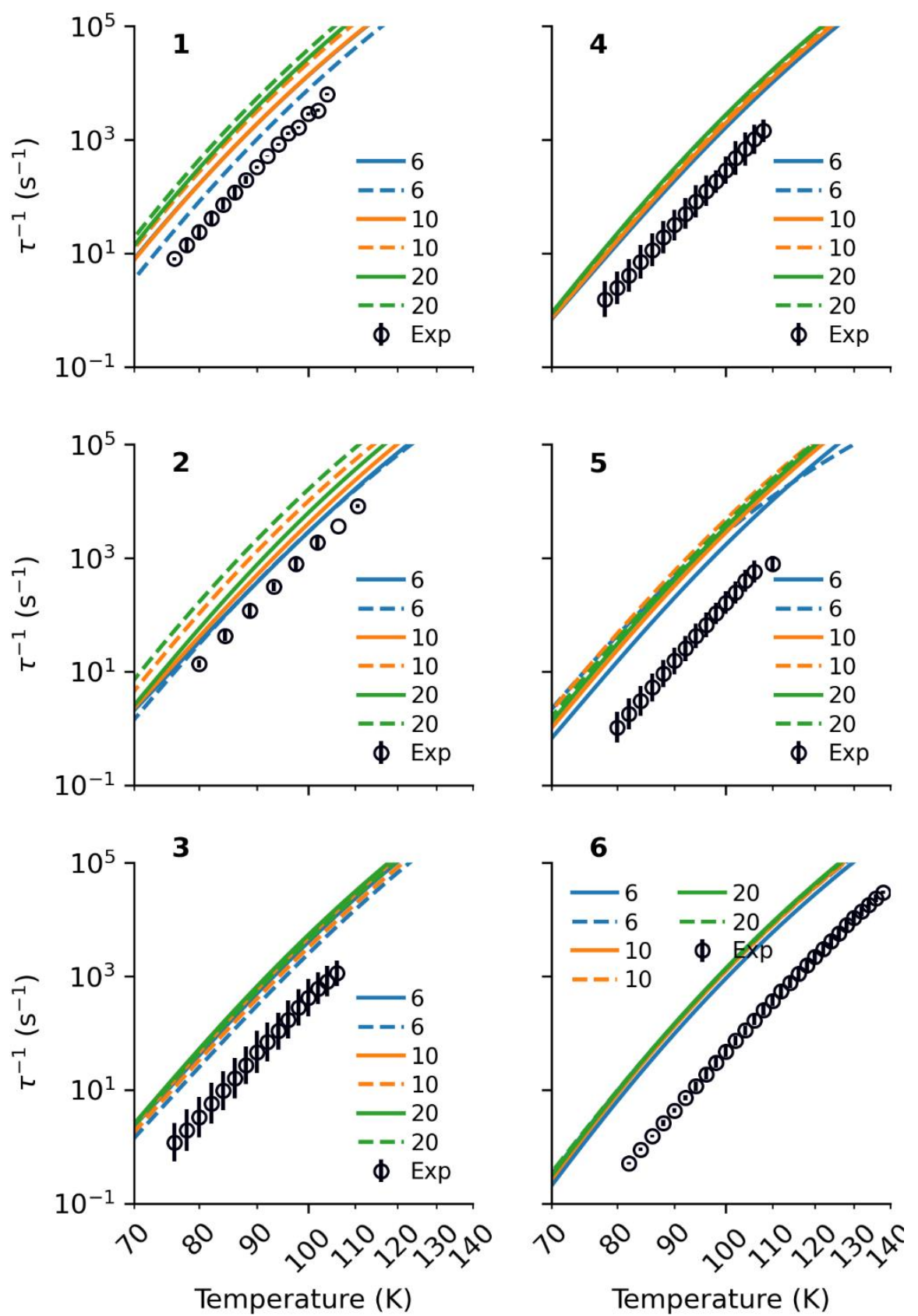

Figure 3. Comparison of experimental (circles) and ab initio calculated (lines, PBE densityfunctional) relaxation rates for 1-6. Solid and dashed lines are obtained without and with IR calibration, respectively. Fixed FWHM linewidths of 6 (blue), 10 (orange) and $20 \mathrm{~cm}^{-1}$ (green) are employed. Experimental error bars are estimated standard deviations derived from the generalised Debye model. ${ }^{24}$ Note: solid blue line for compound $\mathbf{1}$ is obscured by the solid orange line. 

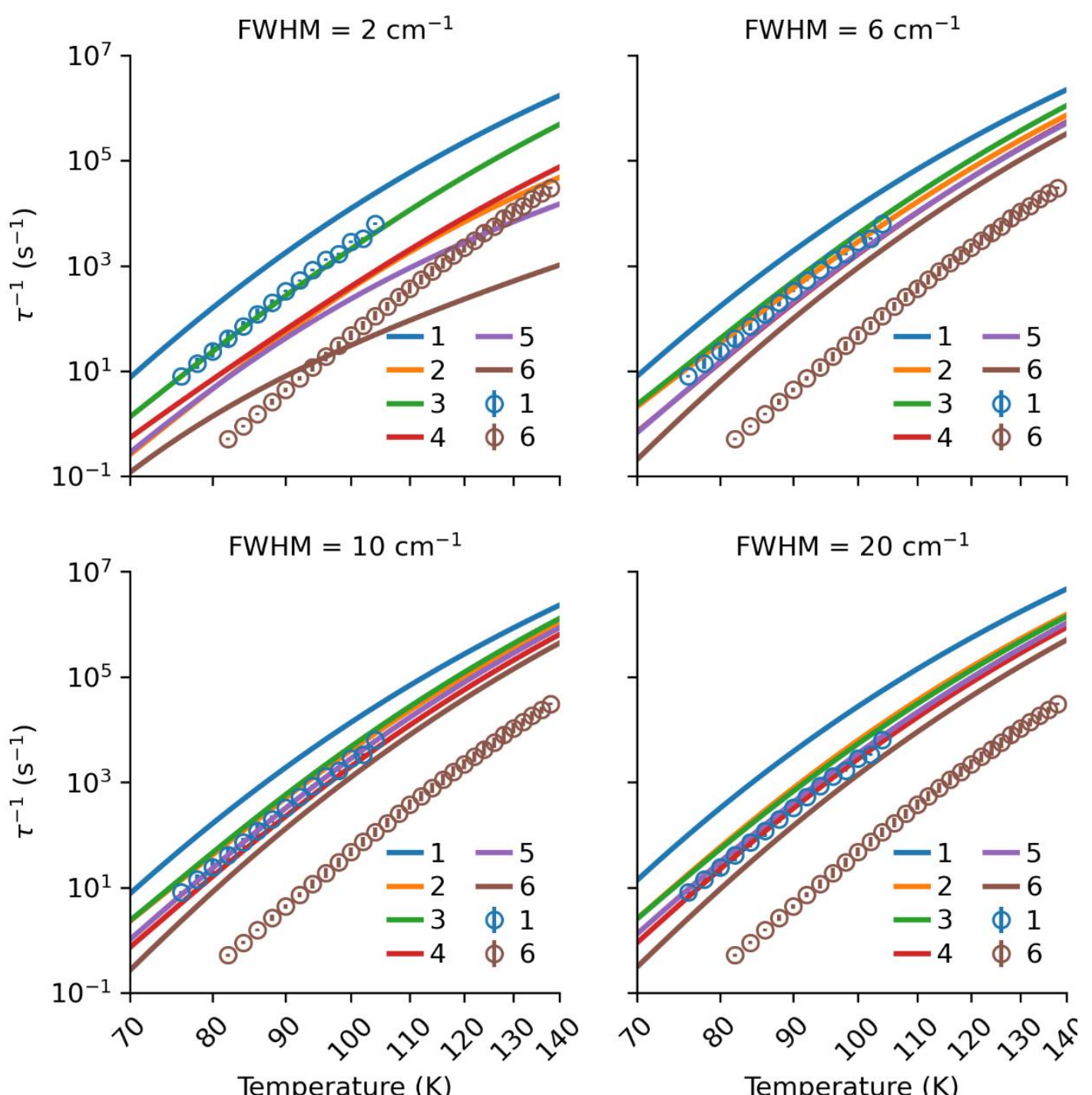

Temperature $(\mathrm{K})$

Temperature $(\mathrm{K})$

Figure 4. Comparison of calculated rates for 1-6, obtained without IR calibration using the PBE density-functional. Experimental data for $\mathbf{1}$ and $\mathbf{6}$ is shown in circles. Experimental error bars are estimated standard deviations derived from the generalised Debye model. ${ }^{24}$

\section{Discussion}

With the results from our $a b$ initio spin-dynamics calculations in hand, and confidence that the relative relaxation rates of 1-6 are well described, we are now in a position to investigate the differences in magnetic relaxation between these compounds, and specifically why it is so slow for 6. This is challenging because the calculated magnetic relaxation rate at a given temperature is the smallest magnitude non-zero eigenvalue of the $16 \times 16$ rate matrix, $\overline{\bar{\gamma}}$, and there is no analytical solution that maps the matrix elements of $\overline{\bar{\gamma}}$ onto its eigenvalues. In our first publication on this topic, we simply considered that the "first step in magnetic relaxation" (the $| \pm 15 / 2\rangle \rightarrow| \pm 13 / 2\rangle$ elements of $\overline{\bar{\gamma}}$ ) would be most important, ${ }^{19}$ but later found that this is not always the most probable first step in relaxation across different Dy(III) SMMs. ${ }^{25,21}$ Our 
second approach was to employ a "knockout" procedure, where the various $| \pm 15 / 2\rangle \rightarrow| \pm x\rangle$ elements of $\overline{\bar{\gamma}}$ are set to zero one-by-one, and the transition responsible for the largest reduction to the overall relaxation rate when removed is determined. ${ }^{21}$ While this gave useful information for bis-alkoxide complexes, ${ }^{21}$ performing this analysis here (extending to all elements of $\overline{\bar{\gamma}}$, not just those starting in $| \pm 15 / 2\rangle)$ shows that no single element of $\overline{\bar{\gamma}}$ has a decisive effect on the overall relaxation rates for any of 1-6 (Figure S25 - S31). Furthermore, analysing the spin phonon coupling strength ${ }^{34}$ for all available modes and comparing them to the electronic energy gaps of 1-6 does not provide a clear answer either (Figure S32-S37).

Therefore, we have devised a new method for unpicking the differences in the relaxation dynamics between these molecules, and herein we focus on the differences between $\mathbf{1}$ and $\mathbf{6}$ which show the greatest disparity in their properties. Firstly, we compare the $\overline{\bar{\gamma}}$ matrices between the two compounds to assess which transitions are different. The difference map of $\log _{10}\left[\overline{\bar{\gamma}}_{6}\right]-\log _{10}\left[\overline{\bar{\gamma}}_{1}\right]$ (Figure S38) shows that the average difference between the lowertriangular elements is $10^{0.7} \approx 5$ times slower for $\mathbf{6}$ than $\mathbf{1}$, in reasonable correlation with the overall magnetic relaxation rates (calculated to be 11 times slower). However, there is a significant spread of differences, and indeed some intriguing features such as elements in $\overline{\bar{\gamma}}_{6}$ that are $10^{10}$ times faster than in $\overline{\bar{\gamma}}_{1}$ (dark purple square). Whilst comparing individual elements of $\overline{\bar{\gamma}}$ provides some insight, there is no clear answer as to the root-cause of these differences. This is because each element in $\overline{\bar{\gamma}}$ is the sum over all vibrational modes (273 and 237 modes for $\mathbf{1}$ and 6, respectively) of the product (Eqn. 1) of the spin-phonon coupling $\left|\left\langle f\left|\widehat{H}_{\mathrm{SP}_{j}}\right| i\right\rangle\right|^{2}$, which reports on how strongly vibrational mode $j$ couples electronic states $i$ and $f$ ), the vibrational occupation $\left|\left\langle n_{j}-1\left|Q_{j}\right| n_{j}\right\rangle\right|^{2}$ or $\left|\left\langle n_{j}+1\left|Q_{j}\right| n_{j}\right\rangle\right|^{2}$, which is the probability of absorption or emission of a vibrational quantum, respectively), and the vibrational density of states (DOS, $\rho_{j}\left(\left|E_{f}-E_{i}\right|\right)$, which reports the proximity of the vibrational mode energy $\hbar \omega_{j}$ to the electronic transition). 


$$
\begin{gathered}
\gamma_{f i}=\sum_{j}^{3 N-6} \gamma_{f i, j} \\
= \begin{cases}\sum_{j}^{3 N-6} \frac{2 \pi}{\hbar}\left|\left\langle f\left|\widehat{H}_{\mathrm{SP}_{j}}\right| i\right\rangle\right|^{2}\left|\left\langle n_{j}-1\left|Q_{j}\right| n_{j}\right\rangle\right|^{2} \rho_{j}\left(\left|E_{f}-E_{i}\right|\right) & E_{f}>E_{i} \\
\sum_{j}^{3 N-6} \frac{2 \pi}{\hbar}\left|\left\langle f\left|\widehat{H}_{\mathrm{SP}_{j}}\right| i\right\rangle\right|^{2}\left|\left\langle n_{j}+1\left|Q_{j}\right| n_{j}\right\rangle\right|^{2} \rho_{j}\left(\left|E_{f}-E_{i}\right|\right) & E_{f}<E_{i}\end{cases}
\end{gathered}
$$

Although every transition is the sum over all modes, due to the conservation of energy and relatively sharp vibrational DOS ( $\rho_{j}$ is a Gaussian function centered at $\hbar \omega_{j}$ with FWHM discussed above), between one and four vibrational modes tend to dominate any given element of $\overline{\bar{\gamma}}$. Thus, we calculate the mode-weighted spin-phonon coupling $\left(\left\langle\widehat{H}_{\mathrm{SP}}\right\rangle_{f i}\right)$, vibrational occupation $\left(\left\langle Q_{j}\right\rangle_{f i}\right)$, and vibrational DOS $\left(\langle\rho\rangle_{f i}\right)$ for each element of $\overline{\bar{\gamma}}$ (Eqns. $\left.2-4\right)$; the effective number of modes associated with each transition $\left(\langle n\rangle_{f i}\right)$ can then be determined simply (Eqn. 5). Hence, the total rate matrix $\overline{\bar{\gamma}}$ can be exactly decomposed into matrix representations of each component (Eqn. 6), where $\circ$ indicates the element-wise (Hadamard) product. This decomposition allows us to pick-and-mix the individual components of the relaxation rate matrix from any compound in order to generate a fictional relaxation rate matrix $\overline{\bar{\gamma}}_{\text {fict }}$, and hence assess the contributing factors to the overall magnetic relaxation rates after diagonalization.

$$
\begin{aligned}
&\left\langle\widehat{H}_{\mathrm{SP}}\right\rangle_{f i}=\sum_{j}^{3 N-6} \frac{\gamma_{f i, j}}{\gamma_{f i}}\left|\left\langle f\left|\widehat{H}_{\mathrm{SP}_{j}}\right| i\right\rangle\right|^{2} \begin{cases}\sum_{j}^{3 N-6} \frac{\gamma_{f i, j}}{\gamma_{f i}}\left|\left\langle n_{j}-1\left|Q_{j}\right| n_{j}\right\rangle\right|^{2} & E_{f}>E_{i} \\
\sum_{j}^{3 N-6} \frac{\gamma_{f i, j}}{\gamma_{f i}}\left|\left\langle n_{j}+1\left|Q_{j}\right| n_{j}\right\rangle\right|^{2} & E_{i}>E_{f}\end{cases} \\
&\langle\rho\rangle_{f i}=\sum_{j}^{3 N-6} \frac{\gamma_{f i, j}}{\gamma_{f i}} \rho_{j}\left(\left|E_{f}-E_{i}\right|\right) \\
&\langle n\rangle_{f i}=\frac{\gamma_{f i}}{2 \pi}\left\langle\widehat{H}_{\mathrm{SP}}\right\rangle_{f i}\langle Q\rangle_{f i}\langle\rho\rangle_{f i}
\end{aligned}
$$




$$
\overline{\bar{\gamma}}=\frac{2 \pi}{\hbar}\left\langle\overline{\bar{H}}_{\mathrm{SP}}\right\rangle \circ\langle\overline{\bar{Q}}\rangle \circ\langle\overline{\bar{\rho}}\rangle \circ\langle\overline{\bar{n}}\rangle
$$

Starting from a base $\overline{\bar{\gamma}}$ matrix of either $\mathbf{1}$ or $\mathbf{6}$, the simplest test is to swap out the individual components one-by-one and determine the relaxation rates of $\overline{\bar{\gamma}}_{\text {fict }}$ (Table 1). We find that by swapping either the spin-phonon coupling, the vibrational DOS or the effective number of modes, the relaxation rates are only altered by a factor of $1-3$ times faster or slower (but note some of these shifts are counterintuitive, owing to the non-trivial relationship between matrix elements and eigenvalues). However, when we swap the vibrational occupation between the two molecules, relaxation in $\mathbf{6}$ becomes 12 times faster, and relaxation in $\mathbf{1}$ becomes 10 times slower. Because magnetic relaxation in the Orbach regime depends on absorption of vibrational quanta, which must be near-resonant with the CF energy gaps (Eqn. 4), the dominance of vibrational occupation found here is direct evidence that the main discriminant in relaxation dynamics between best-in-class $\mathbf{6}$ versus worst-in-class $\mathbf{1}$ is the size the CF splitting, as previously suggested. ${ }^{23}$

Table 1. Breakdown of relaxation rates between $\mathbf{1}$ and $\mathbf{6}$ via a mode-averaging procedure. Relaxation rates are calculated using the PBE density-functional, without IR calibration, at 100 $\mathrm{K}$ with $\mathrm{FWHM}=10 \mathrm{~cm}^{-1}$. Top portion corresponds to a base $\overline{\bar{\gamma}}$ matrix of $\mathbf{1}$, while bottom portion corresponds to a base matrix of $\mathbf{6}$. Rows are ordered by increasing rate.

\begin{tabular}{|c|c|c|c|c|c|}
\hline$\left\langle\overline{\bar{H}}_{\mathrm{SP}}\right\rangle$ & $\langle\overline{\bar{Q}}\rangle$ & $\langle\overline{\bar{\rho}}\rangle$ & $\langle\overline{\bar{n}}\rangle$ & $\tau^{-1}$ & $\tau^{-1 / \tau^{-1}}$ \\
\hline $\mathbf{1}$ & $\mathbf{6}$ & $\mathbf{1}$ & $\mathbf{1}$ & $1.32 \times 10^{3}$ & 0.10 \\
\hline $\mathbf{1}$ & $\mathbf{1}$ & $\mathbf{1}$ & $\mathbf{1}$ & $1.37 \times 10^{4}$ & 1 \\
\hline $\mathbf{6}$ & $\mathbf{1}$ & $\mathbf{1}$ & $\mathbf{1}$ & $142 \times 10^{4}$ & 1.04 \\
\hline $\mathbf{1}$ & $\mathbf{1}$ & $\mathbf{1}$ & $\mathbf{6}$ & $1.80 \times 10^{4}$ & 1.31 \\
\hline $\mathbf{1}$ & $\mathbf{1}$ & $\mathbf{6}$ & $\mathbf{1}$ & $3.27 \times 10^{4}$ & 2.39 \\
\hline$\left\langle\overline{\bar{H}}_{\mathrm{SP}}\right\rangle$ & $\langle\overline{\bar{Q}}\rangle$ & $\langle\overline{\bar{\rho}}\rangle$ & $\langle\overline{\bar{n}}\rangle$ & $\tau^{-1}$ & $\tau^{-1} / \tau^{-1}$ \\
\hline $\mathbf{6}$ & $\mathbf{6}$ & $\mathbf{6}$ & $\mathbf{1}$ & $9.71 \times 10^{2}$ & 0.78 \\
\hline $\mathbf{6}$ & $\mathbf{6}$ & $\mathbf{6}$ & $\mathbf{6}$ & $1.25 \times 10^{3}$ & 1 \\
\hline $\mathbf{6}$ & $\mathbf{6}$ & $\mathbf{1}$ & $\mathbf{6}$ & $1.43 \times 10^{3}$ & 1.14 \\
\hline $\mathbf{1}$ & $\mathbf{6}$ & $\mathbf{6}$ & $\mathbf{6}$ & $4.52 \times 10^{3}$ & 3.62 \\
\hline $\mathbf{6}$ & $\mathbf{1}$ & $\mathbf{6}$ & $\mathbf{6}$ & $1.52 \times 10^{4}$ & 12.16 \\
\hline
\end{tabular}


In order to assess whether the original proposal for removal of the $\mathrm{C}-\mathrm{H}$ groups in $\mathbf{2}$ in order to improve magnetic memory (i.e. engineering the spin-phonon coupling) is indeed behind the increased performance of the $\mathbf{6}$ (calculated to be 1.4 times slower than 2 at $100 \mathrm{~K}$ ), or if the changes are simply due to an increased CF splitting as it is for $\mathbf{1}$, we have performed the mode-weighted analysis comparing 2 with 6 (Table S11). Starting from the base $\overline{\bar{\gamma}}$ matrix of $\mathbf{2}$ and swapping the vibrational occupation component for that found in $\mathbf{6}$ decreases the rate by a factor of 5.3, and swapping out the spin-phonon coupling decreases the rate by a factor of 1.3, while swapping out the vibrational DOS or the effective number of modes from 6 actually increase the rate by factors of 1.1 and 1.5, respectively (and vice versa, the inverse is true). Hence, it seems that both an increased CF splitting in $\mathbf{6}$ (via the vibrational occupation terms) and a reduced spin-phonon coupling are responsible for slowing down relaxation in $\mathbf{6}$ compared to $\mathbf{2}$, but that the former effect is dominant. Hence, this analysis suggests that the enhancements achieved in slowing magnetic relaxation in $\left[\mathrm{Dy}\left(\mathrm{Cp}^{\mathrm{R}}\right)_{2}\right]^{+}$cations has not come about via engineering the spin-phonon coupling, but rather by enlarging the $\mathrm{CF}$ splitting.

To explore how far performance of Dy(III) SMMs can be enhanced, we have made a selection of homoleptic bis-persubstituted-aromatic sandwich complexes of the $\left[\mathrm{Dy}\left(\mathrm{C}_{5} \mathrm{R}_{5}\right)_{2}\right]^{+}$ $(\mathrm{R}=\mathrm{H}, \mathrm{Me})$ and $\left[\mathrm{Dy}\left(\mathrm{C}_{4} \mathrm{R}_{4}\right)_{2}\right]^{-}\left(\mathrm{C}_{4} \mathrm{R}_{4}\right.$ is a persubstituted cyclobutadienyl dianion, $\mathrm{R}=\mathrm{H}, \mathrm{Me}$, ${ }^{\mathrm{i}} \mathrm{Pr},{ }^{\mathrm{t}} \mathrm{Bu}$ ) varieties, in addition to three proposed SMM candidates from the literature (viz. $\left[\mathrm{Dy}\left(\mathrm{C}_{5} \mathrm{I}_{5}\right)_{2}\right]^{+},{ }^{20}\left\{\right.$ DyFlourene $\left.{ }^{\mathrm{i}} \mathrm{Pr}\right\}=\left[\mathrm{Dy}(3,6,9 \text {-tri-iso-propyl-flourenide })_{2}\right]^{+},{ }^{20}$ and $\left[\mathrm{Dy}\left(\mathrm{N}_{5}\right)_{2}\right]^{+}$ ${ }^{35}$ ), and used our ab initio spin-dynamics methodology to predict their magnetic relaxation rates (Figure 5a). Compared to references 20 and 35, here we have performed a full spin-dynamics calculation to arrive at predicted magnetic relaxation rates, rather than assessing the spinphonon coupling and/or electronic states alone. This allows us to predict that $\left[\mathrm{Dy}\left(\mathrm{C}_{5} \mathrm{I}_{5}\right)_{2}\right]^{+}$ would have relaxation rates 1-2 orders of magnitude faster than $\mathbf{6}$, and $\left\{\right.$ DyFlourene $\left.{ }^{i} \operatorname{Pr}\right\}$ would be 3-6 orders of magnitude faster than $\mathbf{6}$. Hence, these results broadly confirm the analysis of Ullah et al., who concluded that $\left[\mathrm{Dy}\left(\mathrm{C}_{5} \mathrm{I}_{5}\right)_{2}\right]^{+}$would be a good SMM and that $\{\mathrm{DyFlourene} \operatorname{Pr}\}$ would not be a good SMM, however we doubt whether $\left[\mathrm{Dy}\left(\mathrm{C}_{5} \mathrm{I}_{5}\right)_{2}\right]^{+}$would surpass the performance of $\mathbf{6}$ based on our results. Following a different strategy, Kotrle and Herchel proposed a series of inorganic sandwich complexes, predicting $\left[\mathrm{Dy}\left(\mathrm{N}_{5}\right)_{2}\right]^{+}$to be a good SMM candidate with $U_{\text {eff }}=1475 \mathrm{~K} \cdot{ }^{35}$ Using our methodology, we find that $\left[\mathrm{Dy}\left(\mathrm{N}_{5}\right)_{2}\right]^{+}$would indeed have a significant energy barrier to relaxation, $U_{\text {eff }}=1292 \mathrm{~K}$ with $\tau_{0}=6.43 \times 10^{-12} \mathrm{~s}$ (the difference in predicted energy barrier is likely due to our use of CASSCF-SO vs. the inclusion of dynamic correlation in ref. 35), but that its relaxation dynamics are 2-4 orders of magnitude faster than for $\mathbf{6}$. 
Examining the cyclobutadienyl and cyclopendadienyl compounds, we find that all dianionic cyclobutadienyl ligand sets generate a total splitting of the $J= \pm 15 / 2$ multiplet that is equal to or larger than compound 6 with two monoanionic $\mathrm{Cp}^{\mathrm{R}}$ ligands, but interestingly, only $\left[\mathrm{Dy}\left(\mathrm{C}_{4}{ }^{\mathrm{t}} \mathrm{Bu}_{4}\right)_{2}\right]^{-}$shows a comparable gap between the ground and first excited doublets (Figures $5 \mathrm{~b}$ and $5 \mathrm{c}$ ); thus, it seems that while dianionic ligands do generally increase the CF splitting, the effect is non-trivial when considering individual $m_{J}$ components. Indeed, we find that $\left[\mathrm{Dy}\left(\mathrm{C}_{4}{ }^{\mathrm{t}} \mathrm{Bu}_{4}\right)_{2}\right]^{-}$has a very similar relaxation rate to $\mathbf{6}$, but that both $\left[\mathrm{Dy}\left(\mathrm{C}_{4} \mathrm{H}_{4}\right)_{2}\right]^{-}$and $\left[\mathrm{Dy}\left(\mathrm{C}_{5} \mathrm{Me}_{5}\right)_{2}\right]^{+}$, which have smaller energy gaps between the ground and first excited doublets, show relaxation rates orders of magnitude smaller than $\mathbf{6}$; all other compounds examined here are predicted to have faster relaxation than $\mathbf{6}$. Fitting the calculated relaxation rates above 100 $\mathrm{K}$ to an Arrhenius law for the Orbach mechanism shows that the predicted $U_{\text {eff }}$ barriers are a maximum of around $2100 \mathrm{~K}$ for this class of compound (Table S12): specifically for the two compounds predicted to have slower relaxation than 6, we find $U_{\text {eff }}=2093 \mathrm{~K}$ and $\tau_{0}=2.48 \times 10^{-}$ ${ }^{11} \mathrm{~s}$ for $\left[\mathrm{Dy}\left(\mathrm{C}_{4} \mathrm{H}_{4}\right)_{2}\right]^{-}$and $U_{\text {eff }}=1549 \mathrm{~K}$ and $\tau_{0}=8.90 \times 10^{-8} \mathrm{~s}$ for $\left[\mathrm{Dy}\left(\mathrm{C}_{5} \mathrm{Me}_{5}\right)_{2}\right]^{+}$, compared to $U_{\text {eff }}=2048 \mathrm{~K}$ and $\tau_{0}=1.03 \times 10^{-12} \mathrm{~s}$ for $6\left(c f . U_{\text {eff }}=2217(16) \mathrm{K}\right.$ and $\tau_{0}=4.2(6) \times 10^{-12} \mathrm{~s}$ found experimentally $\left.{ }^{23}\right)$. While $\left[\mathrm{Dy}\left(\mathrm{C}_{4} \mathrm{H}_{4}\right)_{2}\right]^{-}$has a similar $U_{\text {eff }}$ barrier to $\mathbf{6}$, the $\tau_{0}$ pre-factor is an order of magnitude larger and hence its relaxation is an order of magnitude slower. Analysis using a mode-weighted decomposition (Table S13) shows that the phonon DOS is the dominant term leading to a slower relaxation rate in $\left[\mathrm{Dy}\left(\mathrm{C}_{4} \mathrm{H}_{4}\right)_{2}\right]^{-}$as compared to 6 . For $\left[\mathrm{Dy}\left(\mathrm{C}_{5} \mathrm{Me}_{5}\right)_{2}\right]^{+}$,

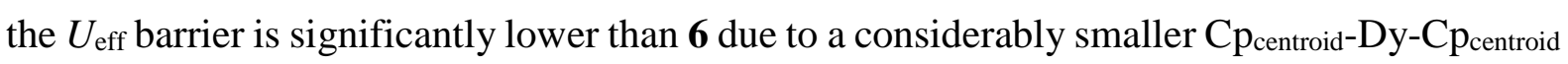
angle of $144^{\circ}$ vs. $160^{\circ}$ (and despite shorter Dy-C $p_{\text {centroid }}$ distances, Table S12), however, the $\tau_{0}$ pre-factor is four orders of magnitude larger than for 6: this is clearly the decisive difference in the relaxation dynamics. Using a mode-weighted decomposition, we again find that the phonon DOS is the origin of the far larger $\tau_{0}$ in $\left[\mathrm{Dy}\left(\mathrm{C}_{5} \mathrm{Me}_{5}\right)_{2}\right]^{+}$than for 6 (Table 2). For both $\left[\mathrm{Dy}\left(\mathrm{C}_{4} \mathrm{H}_{4}\right)_{2}\right]^{-}$and $\left[\mathrm{Dy}\left(\mathrm{C}_{5} \mathrm{Me}_{5}\right)_{2}\right]^{+}$this is confirmed by comparing the vibrational mode distributions with the electronic energy levels (Figures S39 and S40 cf. S37), showing that in addition to there being far fewer vibrational modes than in $\mathbf{6}$, they are also less frequently onresonance with electronic transitions. Hence, it seems that while $U_{\text {eff }}$ barriers may have reached their limit in such sandwich compounds, engineering molecular vibrational modes can play a significant role in increasing the relaxation times of SMMs. 

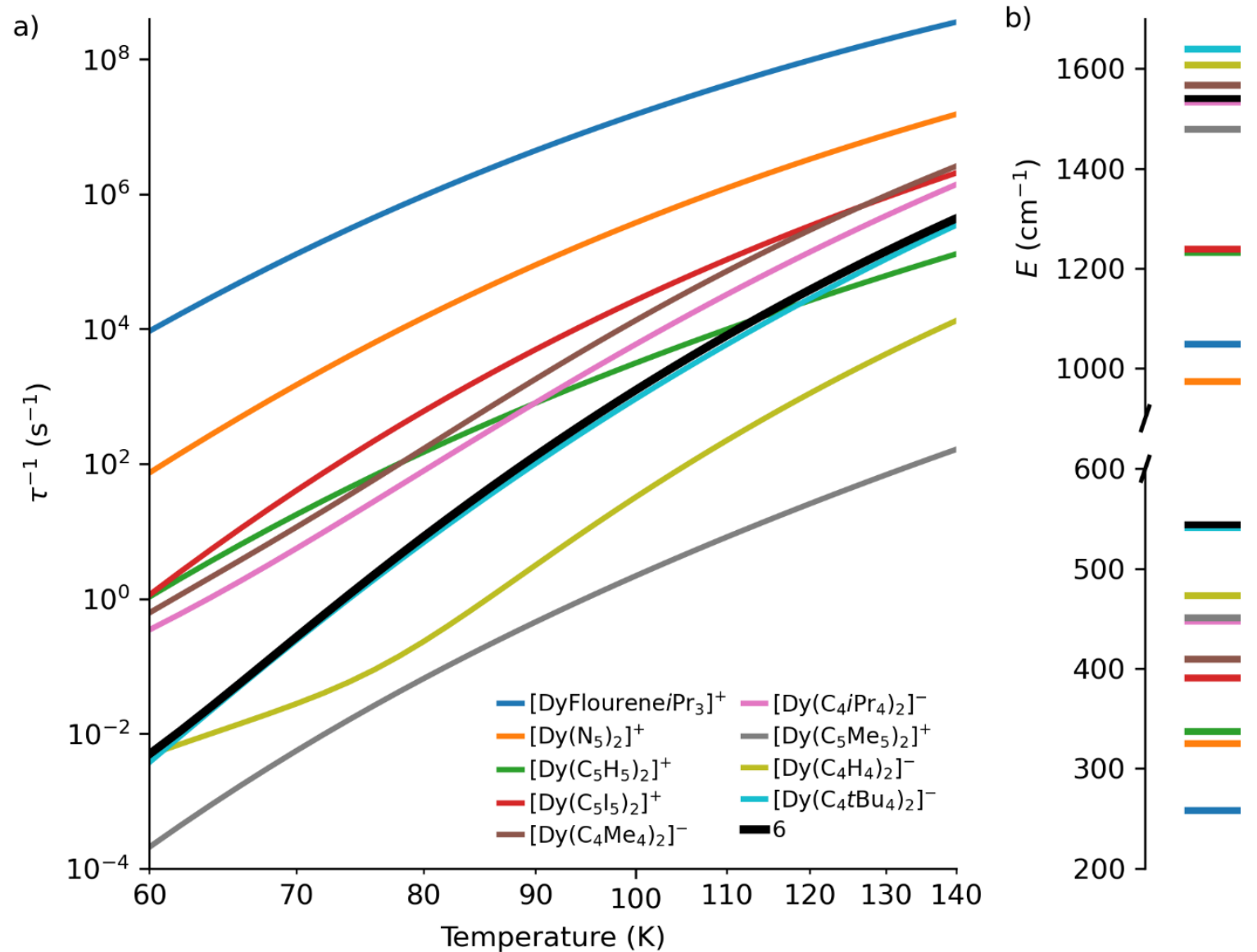

Figure 5. (a) Comparison of calculated rates for theoretical SMMs compared to 6, obtained using the PBE density-functional. (b) Energy of highest (top) and first excited (bottom) doublet in the ${ }^{6} \mathrm{H}_{15 / 2}$ multiplet. 
Table 2. Breakdown of relaxation rates between $\left[\mathrm{Dy}\left(\mathrm{C}_{5} \mathrm{Me}_{5}\right)_{2}\right]^{+}$and $\mathbf{6}$ via a mode-weighting procedure. Relaxation rates are calculated using the PBE density-functional, without calibration, at $100 \mathrm{~K}$ with $\mathrm{FWHM}=10 \mathrm{~cm}^{-1}$. Top portion corresponds to a base $\overline{\bar{\gamma}}$ matrix of $\left[\mathrm{Dy}\left(\mathrm{C}_{5} \mathrm{Me}_{5}\right)_{2}\right]^{+}$, while bottom portion corresponds to a base matrix of $\mathbf{6}$. Rows are ordered by increasing rate.

\begin{tabular}{|c|c|c|c|c|c|}
\hline$\left\langle\overline{\bar{H}}_{\mathrm{SP}}\right\rangle$ & $\langle\overline{\bar{Q}}\rangle$ & $\langle\overline{\bar{\rho}}\rangle$ & $\langle\overline{\bar{n}}\rangle$ & $\tau^{-1}$ & $\tau^{-1} / \tau^{-1} \mathrm{Dy}_{\left.\left(\mathrm{C}_{5} \mathrm{Me}_{5}\right)_{2}\right]^{+}}$ \\
\hline$\left[\mathrm{Dy}\left(\mathrm{C}_{5} \mathrm{Me}_{5}\right)_{2}\right]^{+}$ & $\mathbf{6}$ & {$\left[\mathrm{Dy}\left(\mathrm{C}_{5} \mathrm{Me}_{5}\right)_{2}\right]^{+}$} & {$\left[\mathrm{Dy}\left(\mathrm{C}_{5} \mathrm{Me}_{5}\right)_{2}\right]^{+}$} & $1.48 \times 10^{0}$ & 0.01 \\
\hline$\left[\mathrm{Dy}\left(\mathrm{C}_{5} \mathrm{Me}_{5}\right)_{2}\right]^{+}$ & {$\left[\mathrm{Dy}\left(\mathrm{C}_{5} \mathrm{Me}_{5}\right)_{2}\right]^{+}$} & {$\left[\mathrm{Dy}\left(\mathrm{C}_{5} \mathrm{Me}_{5}\right)_{2}\right]^{+}$} & $\mathbf{6}$ & $2.37 \times 10^{0}$ & 0.01 \\
\hline $\mathbf{6}$ & {$\left[\mathrm{Dy}\left(\mathrm{C}_{5} \mathrm{Me}_{5}\right)_{2}\right]^{+}$} & {$\left[\mathrm{Dy}\left(\mathrm{C}_{5} \mathrm{Me}_{5}\right)_{2}\right]^{+}$} & {$\left[\mathrm{Dy}\left(\mathrm{C}_{5} \mathrm{Me}_{5}\right)_{2}\right]^{+}$} & $5.02 \times 10^{0}$ & 0.02 \\
\hline$\left[\mathrm{Dy}\left(\mathrm{C}_{5} \mathrm{Me}_{5}\right)_{2}\right]^{+}$ & {$\left[\mathrm{Dy}\left(\mathrm{C}_{5} \mathrm{Me}_{5}\right)_{2}\right]^{+}$} & {$\left[\mathrm{Dy}\left(\mathrm{C}_{5} \mathrm{Me}_{5}\right)_{2}\right]^{+}$} & {$\left[\mathrm{Dy}\left(\mathrm{C}_{5} \mathrm{Me}_{5}\right)_{2}\right]^{+}$} & $2.19 \times 10^{2}$ & 1 \\
\hline$\left[\mathrm{Dy}\left(\mathrm{C}_{5} \mathrm{Me}_{5}\right)_{2}\right]^{+}$ & {$\left[\mathrm{Dy}\left(\mathrm{C}_{5} \mathrm{Me}_{5}\right)_{2}\right]^{+}$} & $\mathbf{6}$ & {$\left[\mathrm{Dy}\left(\mathrm{C}_{5} \mathrm{Me}_{5}\right)_{2}\right]^{+}$} & $1.83 \times 10^{3}$ & 8.36 \\
\hline$\left\langle\overline{\bar{H}}_{\mathrm{SP}}\right\rangle$ & $\langle\overline{\bar{Q}}\rangle$ & $\langle\overline{\bar{\rho}}\rangle$ & $\langle\overline{\bar{n}}\rangle$ & $\tau^{-1}$ & $\tau^{-1} / \tau^{-1} 6$ \\
\hline $\mathbf{6}$ & $\mathbf{6}$ & {$\left[\mathrm{Dy}\left(\mathrm{C}_{5} \mathrm{Me}_{5}\right)_{2}\right]^{+}$} & $\mathbf{6}$ & $3.35 \times 10^{0}$ & 0.003 \\
\hline$\left[\mathrm{Cy}\left(\mathrm{C}_{5} \mathrm{Me}_{5}\right)_{2}\right]^{+}$ & $\mathbf{6}$ & $\mathbf{6}$ & {$\left[\mathrm{Dy}\left(\mathrm{C}_{5} \mathrm{Me}_{5}\right)_{2}\right]^{+}$} & $9.24 \times 10^{2}$ & 0.74 \\
\hline $\mathbf{6}$ & $\mathbf{6}$ & $\mathbf{6}$ & $\mathbf{6}$ & $9.57 \times 10^{2}$ & 0.77 \\
\hline $\mathbf{6}$ & {$\left[\mathrm{Dy}\left(\mathrm{C}_{5} \mathrm{Me}_{5}\right)_{2}\right]^{+}$} & $\mathbf{6}$ & $\mathbf{6}$ & $1.25 \times 10^{3}$ & 1 \\
\hline
\end{tabular}

\section{Conclusion}

Design criteria for increasing magnetic anisotropy in Dy(III)-based SMMs have been produced and verified, leading to dramatic increases in effective energy barriers to magnetic relaxation and vast improvements in SMM performance. However, the route towards further improvements is unclear. By developing an $a b$ initio methodology for calculating spindynamics with relative quantitative accuracy, along with a new analysis technique, we are now able to probe the origins of differing SMM performance directly. This has allowed us to prove that the current best-performing $\mathrm{SMM}\left[\mathrm{Dy}\left(\mathrm{Cp}^{\mathrm{iPr} 5}\right)\left(\mathrm{Cp}^{*}\right)\right]\left[\mathrm{B}\left(\mathrm{C}_{6} \mathrm{~F}_{5}\right)_{4}\right](\mathbf{6})$ is better than both the worst in its class $\left[\mathrm{Dy}\left(\mathrm{Cp}^{\mathrm{iPr} 4}\right)_{2}\right]\left[\mathrm{B}\left(\mathrm{C}_{6} \mathrm{~F}_{5}\right)_{4}\right]$ (1) and the original dysprosocenium SMM $\left[\mathrm{Dy}\left(\mathrm{Cp}^{\mathrm{ttt}}\right)_{2}\right]\left[\mathrm{B}\left(\mathrm{C}_{6} \mathrm{~F}_{5}\right)_{4}\right](2)$ because it has a larger CF splitting. Subsequently, we have predicted that further enhancements to $U_{\text {eff }}$ seem minimal and that progress in slowing magnetic relaxation in the Orbach regime could be obtained by moving vibrational modes off-resonance with electronic transitions, even if $U_{\text {eff }}$ barriers are adversely affected. 


\section{Acknowledgements}

We thank the European Research Council (ERC-2019-STG-851504), EPSRC (PhD scholarship to J.G.C.K.), The Royal Society (University Research Fellowship to N.F.C.) and The University of Manchester (Presidential Fellowship to N.F.C.) for support. We thank Daniel Corbett and the Computational Shared Facility at The University of Manchester for assistance.

\section{References}

1'Sessoli, R. Nature, 548, 400-401 (2017).
${ }^{2}$ Sessoli, R., Gatteschi D., Caneschi, A., \& Novak, M., Nature, 365 (6442), 141-143, (1993).
${ }^{3}$ Orbach, R., Proc. Roy. Soc. A., 264, 458-484, (1961).
${ }^{4}$ Gatteschi, D., Sessoli, R. \& Villain, J., Molecular Nanomagnets. (Oxford University Press, 2006).
${ }^{5}$ Chudnovsky, E. M. \& Tejada, J., Macroscopic Quantum Tunneling of the Magnetic Moment.
(Cambridge University Press, 2005).
6 Bartolomé, J., Luis, F. \& Fernández, J. F., Molecular Magnets. Physics and Applications. (NanoScience and Technology; Springer Berlin Heidelberg: Berlin, Heidelberg, 2014).

${ }^{7}$ Naoto, I., Miki, S., Tadahiko, I., Shin-ya, K. \& Kaizu, Y., J. Am. Chem. Soc., 125 (29), 8694-8695 (2003).

${ }^{8}$ Ishikawa, N., Miki, S., Tomoko, O., Naohiro, T., Tomochika, I. \& Kaizu, Y., Inorg. Chem., 42 (7), 2440-2446 (2003).

${ }^{9}$ Sievers, J., Zeitschrift für Phys. B Condens. Matter, 45 (4), 289-296 (1982).

${ }^{10}$ Rinehart, J. D. \& Long, J. R., Chem. Sci., 2 (11), 2078 (2011).

${ }^{11}$ Chilton, N. F., Collison, D., McInnes, E. J. L., Winpenny R. E. P. \& Soncini A., Nat. Commun., 4, 2551 (2013).

${ }^{12}$ Aravena, D. \& Ruiz, E., Inorg. Chem., 52 (23), 13770-13778 (2013).

${ }^{13}$ Giansiracusa, M. J., Kostopoulos, A. K., Collison, D., Winpenny, R. E. P. \& Chilton, N. F., Chem. Commun., 55, 7025 (2019).

${ }^{14}$ Shrivastava, K. N., Phys. status solidi, 117 (2), 437-458 (1983).

${ }^{15}$ Lunghi, A., Totti, F., Sessoli, R. \& Sanvito, S., Nat. Commun., 8, 14620, (2017).

${ }^{16}$ Lunghi, A., Totti, F., Sanvito, S. \& Sessoli, R., Chem. Sci., 8, 6051-6059 (2017).

${ }^{17}$ Escalera-Moreno, L., Suaud, N., Gaita-Ariño, A. \& Coronado, E., J. Phys. Chem. Lett., 8, 1695-1700, (2017).

${ }^{18}$ Escalera-Moreno, L., Baldoví, J. J., Gaita-Ariño, A. \& Coronado, E., Chem. Sci., 9, 3265-3275, (2018).

${ }^{19}$ Goodwin, C. A. P., Ortu, F., Reta, D., Chilton, N. F. \& Mills, D. P., Nature, 548 (7668), 439-442, (2017).

${ }^{20}$ Ullah, A., Cerdá, J., Baldoví, J. J., Varganov, S. A., Aragó, J. \& Gaita-Ariño, A., Phys. Chem. Lett., 10, 24, 7678-7683, (2019).

${ }^{21}$ Yu, K.-X., Kragskow, J. G. C., Ding, Y.-S., Zhai, Y.-Q., Reta, D., Chilton, N. F. \& Zheng, Y.-Z. Chem, 6, 1777-1793 (2020).

${ }^{22}$ McClain, K. R.; Gould, C. A.; Chakarawet, K.; Teat, S. J.; Groshens, T. J.; Long, J. R.; Harvey, B. G. Chem. Sci., 9, 8492-8503 (2018).

${ }^{23}$ Guo, F.-S.; Day, B. M.; Chen, Y.-C.; Tong, M.-L.; Mansikkamäki, A. \& Layfield, R. A. Science, 362, 1400, (2018).

${ }^{24}$ Reta, D. \& Chilton, N. F., Phys. Chem. Chem. Phys., 21, 23567-23575 (2019). 
${ }^{25}$ Evans, P., Reta, D., Whitehead, G. F.S., Chilton, N. F. \& Mills, D. P. J. Am. Chem. Soc., 141, 19935-19940 (2019).

${ }^{26}$ Perdew, J. P., Burke, K. \& Ernzerhof, M. Phys. Rev. Lett., 77 (18), 3865-3868, (1996).

${ }^{27}$ Perdew, J. P., Burke, K. \& Ernzerhof, M. Phys. Rev. Lett., 78 (7), 1396-1396 (1997).

${ }^{28}$ Adamo, C. \& Varone, V., J. Chem. Phys., 110, 6158-69 (1999).

${ }^{29}$ Grimme, S. Wiley Interdiscip. Rev. Comput. Mol. Sci., 1 (2), 211-228 (2011).

${ }^{30}$ Frisch, M. J. et al. Gaussian 09, Revision D.01, Gaussian, Inc., Wallingford CT, 2016

${ }^{31}$ Aquilante, F. et al. J. Comput. Chem., 37, 506-541 (2016).

${ }^{32}$ Ungur, L. \& Chibotaru, L. F., Chemistry - A European Journal, 23, 3708-3718 (2017).

${ }^{33}$ Ding, Y-S., Han, T., Zhai, Y. Q., Reta, D., Chilton, N. F., Winpenny, R. E. P. \& Zheng, Y. Z., Chem. Eur. J., 26, 5893-5902 (2020).

${ }^{34}$ Chang, N. C., Gruber, J. B., Leavitt, R. P. \& Morrison, C. A., J. Chem. Phys., 76, 3877-3889, (1982).

${ }^{35}$ Kotrle, K. \& Herchel, R. Inorg. Chem., 58, 20, 14046-14057 (2019). 